\section{Generalizing, deleting and distorting information about the experience and communication of chronic pain}
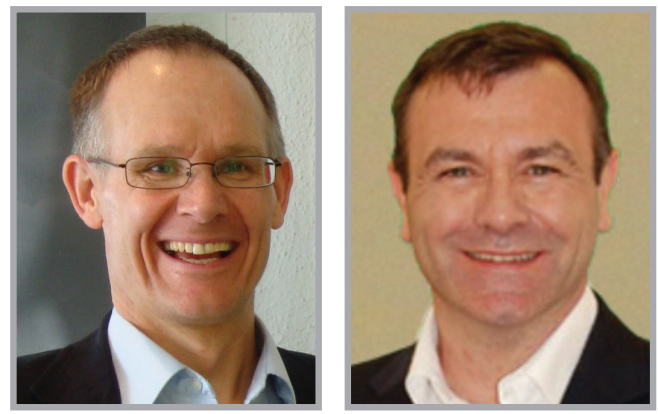

“...Pain practitioners are more likely to establish what pain means to a person if they are able to understand the person's experience and the context within which it is situated."

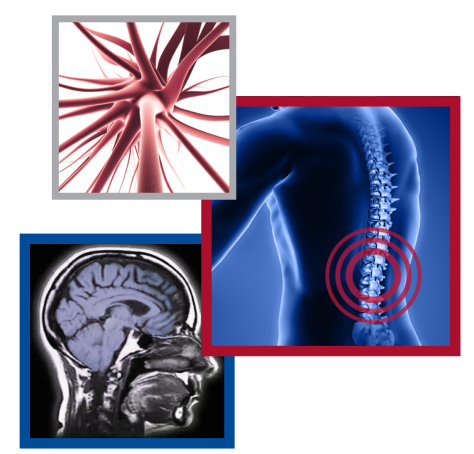

Mark I Johnson ${ }^{* 1}$ \& Matt Hudson²

First draft submitted: 29 June 2016; Accepted for publication: 12 July 2016; Published online: 1 August 2016

There is growing support for a shift in chronic pain management toward a patientcentered approach that accounts for the lived experience of the person. A review of research using interpretative phenomenological analyses of the lived experience of people with chronic pain found that they had challenges understanding their self-identity and sense of moral worth [1]. Disbelief of others that a person has chronic pain results in emotional distress, isolation and stigma that is perceived as a challenge to that person's integrity and identity [2]. A meta-ethnographic synthesis of the findings of qualitative research investigating the experience of people with chronic low back pain has found that people struggle to reconcile the self with their persistent pain revealing a need for further research on pain and social identity [3].

A person's mental map of themselves and their world, including their underlying thinking, affects their final experience of pain. This mental map is reflected in the language used by people in daily conversatives) about their pain experience. In the social sciences narratives are used to study a person's experience of illness, including pain, to provide insights into the social and cultural underpinnings of their physiological reality [4]. Narratives used by people to describe their experience of living with pain help in the understanding of a person's core values and the meaning they attribute to the pain [5]. However, people rarely communicate events and experiences as they actually happen but describe the event by generalizing, deleting and distorting information. Filtering information in this way influences the person's future thinking, core values and health and well-being. The purpose of this editorial is to discuss the influence of generalizing, deleting and distorting information when people describe their experience of persistent pain in relation to communicative frameworks during consultations. tion, including the stories they tell (narra-

'Centre for Pain Research, School of Clinical \& Applied Sciences, Leeds Beckett University, City Campus, Leeds,

\section{KEYWORDS}

- chronic pain - frameworks of communication $\bullet$ neurolinguistic programming

\footnotetext{
"There is growing support for a shift in chronic pain management toward a patient-centered approach that accounts for the lived experience of the person."
} 


\section{“The development of communication frameworks with a patient-centered focus ... are more likely to result in accurate diagnosis of factors influencing pain and disability resulting in \\ pain management solutions tailed to the specific needs and viewpoint of the patient.”}

\section{"The eyes will only affirm or deny what your mind believes."}

- Matt Hudson [6]

The concept of generalizing deleting and distorting information during communication was developed as a central tenet of the meta-model of neurolinguistic programming (NLP). NLP was developed in the 1970 s as a neurological language and behavioral approach to communication, personal development, counseling and psychotherapy [7]. NLP practitioners seek to improve well-being by influencing mental (subjective) representations of experiences and to encourage them to imitate 'healthy skills'. Subjective representations of a person's experiences can be modified through introspection and are expressed through language and behaviors. NLP is popular within sporting and business environments, but is also used as an adjunct in the management of depression, phobias and habit disorders. NLP training is informally regulated and accredited at diploma, practitioner and master practitioner level by The International NLP Trainer's Association and by The Association of NLP in the UK. Sturt et al. [8] reported that spending on NLP training within the National Health Service trusts in the UK was modest and mostly by administrative and managerial staff. NLPbased counseling services have been developed in some National Health Service trusts for weight loss, substance misuse and smoking cessation, although NLP has not been approved by NICE because of a paucity of research on effectiveness for health-related outcomes. Sturt et al. [8] conducted a systematic review that included ten studies on anxiety disorders, weight maintenance, morning sickness, substance misuse and claustrophobia during MRI scanning. They concluded that there was insufficient evidence to support the allocation of resources to NLP therapy outside of research purposes. Five randomized controlled trials were included in the review and four of these randomized controlled trials found no significant difference between NLP and comparison groups. One study found improvements in 'psychological difficulties' compared with a waiting list control [9]. Moreover, there has been criticism of the theoretical underpinning of specific aspects of NLP leading to claims that NLP is pseudoscience [10]. For example, some proponents of NLP claim that there is a relationship between eye movements and thought, although the paucity of experimental research that exists does not support this claim [11,12].

Skepticism of the theoretical underpinning techniques adopted by NLP practitioners coupled with a lack of evidence for clinical effectiveness has resulted in NLP being ignored by mainstream medicine. Pain practitioners use various psychological approaches to help people manage persistent pain with cognitive-behavioral therapy - the foundation of most practice. Cognitive-behavioral therapy is delivered over a series of sessions to develop adaptive cognitive and behavioral pain coping skills, including restructuring of maladaptive cognitions, appropriate goal setting stress management through relaxation, breathing and visual imagery, and effective use of social reinforcement. Recently, however, there has been renewed interest in the use of NLP in primary care settings as part of communication frameworks used to influence thinking and behavior $[8,13]$. We are interested in the use of NLP to explore a person's mental map of themselves and their world. By exploring how underlying thinking affects the mental map, it may provide a novel approach to aid a person's reconceptualization of pain. The foundation of NLP is a pragmatic communications model (the meta-model) suggesting that we do not communicate our experiences faithfully. Rather, communication and thinking is modified through filters that generalize, delete and distort information providing people with 'short cuts' when analyzing incoming information enabling them to survive in the information overload of modern society. However, these filters can limit the view of oneself and the world in which we live.

Persistent pain and suffering affects a person's sense of self by interfering with cognitive, affective and behavioral processes threatening the identity of the person [14]. Self is not a single entity but rather a construction of a variety of aspects of self, including, for example, self in the past, present and future; actual and ideal self; and how others see oneself. This sense of self develops over time from beliefs and values of oneself and others. Persistent pain and the self become enmeshed to create a self-identity of 'pain patient'. Morley [14] provides evidence that people with persistent pain may be susceptible to preferentially respond to certain types of information (cognitive biases) because pain and the self were intertwined. Thus, diagnoses 
and pain management solutions, especially in relation to lifestyle changes, offered by practitioners may be misinterpreted by pain patients as judgments on their beliefs, values and sense of self. These cognitive biases are associated with generalization, deletion and distortion of information related to events and experiences.

Generalization is the process of making general conclusions about an event by attributing the experience of one event to the entire category of which the experience was an example. Generalization is useful because it enables individuals to apply overarching principles to single events and enables rapid adaptation to novel situations. For example, from an early age we know how to open doors because we generalize the outcome of the experience of ourselves and others using doors. Generalization helps to generate beliefs and, therefore, they may be dangerous in certain contexts. For example, the act of one person from a particular group can be generalized to represent the act of all people from that group as seen in racism, sexism or nationalism. Generalization may hamper positive self-beliefs and positive emotional states by establishing rules that are detrimental to health and well-being. For example, generalization may reinforce maladaptive behaviors such as fearavoidance of movement because of the generalization that moving causes pain which causes harm. This generalization is beneficial immediately following a traumatic injury because it will prevent further tissue damage and promote tissue healing. However, the generalization can be disadvantageous for long-term nonspecific chronic musculoskeletal pain where the drivers for pain are not strongly coupled to damage in peripheral tissue. Hence, generalizations may manifest as phobias.

Deletion is the process of filtering out information by omitting details from events through selective attention of certain aspects of an experience. Deletion enables people to focus on the critical aspects of our experiences and to ignore the vast amount of less important information encountered in daily life. However, there is a danger of deleting (ignoring) information that is considered meaningless when it is not and this may impact negatively on the precision and accuracy of information conveyed to oneself and to others. Distortion is the process of misrepresenting incoming sensory information and modifying the meaning, interpretation and description of events and experiences.
Distortion is mediated by cognitive biases where people fail to adequately assess their capabilities, resulting in illusory inferiority or superiority (i.e., Dunning-Kruger effect). The process of distortion influences a person's selfimage where people interpret experiences confirm pre-existing beliefs, even if these beliefs are not necessarily representative of reality. People often have a distorted self-image of themselves and this can reinforce illness and hinder wellbeing. People with long-standing unresolved pain often have a negative self-image of themselves. Thus, cognitive biases associated with generalization, deletion and distortion affect an individual's sense of self and may contribute to self-sabotage whereby thoughts, attitudes and behaviors prevent individuals achieving their desired goals.

Systematic reviews of show that interactions between patients and practitioners during consultations are critical for positive health-related outcomes [15]. Pain practitioners are more likely to establish what pain means to a person if they are able to understand the person's experience and the context within which it is situated. The development of communication frameworks with a patient-centered focus, such as ideas, concerns and expectations [16], are more likely to result in accurate diagnosis of factors influencing pain and disability resulting in pain management solutions tailed to the specific needs and viewpoint of the patient. To date, little attention has been given in research literature to frameworks of communication adopted by NLP practitioners such as cognitive processes that generalize, delete and distort the communication of experiences of pain patients and how this affects the mental map of themselves and their world. We hope that this editorial will catalyze interest in this field.

\section{"Thought defines who you are today and can sabotage who you can be tomorrow." \\ - Matt Hudson [6].}

Financial \& competing interests disclosure

M Hudson is Director of Training at Mind International Training Associates. The authors have no other relevant affiliations or financial involvement with any organization or entity with a financial interest in or financial conflict with the subject matter or materials discussed in the manuscript apart from those disclosed.

No writing assistance was utilized in the production of this manuscript. "...little attention has been given in research literature to frameworks of communication adopted by neurolinguistic programming practitioners such as cognitive processes that generalize, delete and distort the communication of experiences of pain patients and how this affects the mental map of themselves and their world." 


\section{References}

Papers of special note have been highlighted as: - of interest; $\bullet$ of considerable interest

1 Edwards I, Jones M, Thacker M, Swisher LL. The moral experience of the patient with chronic pain: bridging the gap between first and third person ethics. Pain Med. 15(3), 364-378 (2014).

2 Newton BJ, Southall JL, Raphael JH, Ashford RL, Lemarchand K. A narrative review of the impact of disbelief in chronic pain. Pain Manag. Nurs. 14(3), 161-171 (2013).

3 Macneela P, Doyle C, O'gorman D, Ruane N, Mcguire BE. Experiences of chronic low back pain: a meta-ethnography of qualitative research. Health Psychol. Rev. 9(1), 63-82 (2015).

4 Hyden LC. Illness and narrative. Sociol. Health Ill. 19(1), 48-69 (1997).

5 Steihaug S, Malterud K. Stories about bodies: a narrative study on self-understanding and chronic pain. Scand. J. Prim. Health 26(3), 188-192 (2008).

6 Hudson M. The Saboteur Within: The Definitive Guide to Eliminating Self-Sabotage. Amazon Distribution GmbH, Leipzig, Germany, 117 (2011)
- Introduces the concept of self-sabotage through negative patterns of thoughts and behaviors.

7 Bandler R, Grinder J. The Structure of Magic I: A Book About Language and Therapy. Science \& Behavior Books, Palo Alto, CA, USA (1975).

8 Sturt J, Ali S, Robertson W et al. Neurolinguistic programming: a systematic review of the effects on health outcomes. Br. J. Gen. Pract. 62(604), e757-e764 (2012).

- One of the few systematic reviews of the effects of neurolinguistic programming on health outcomes.

9 Stipancic M, Renner W, Schutz P, Dond R. Effects of neuro-linguistic psychotherapy on psychological difficulties and perceived quality of life. Couns. Psychother. Res. 10(1), 39-49 (2010).

10 Heap M. The validity of some early claims of neuro-linguistic programming. Skeptical Intelligence 11, 6-13 (2008).

11 Sharpley C. Research findings on neurolinguistic programming: nonsupportive data or an untestable theory? J. Couns. Psychol. 34, 103-107 (1987).
12 Wiseman R, Watt C, Ten Brinke L, Porter S, Couper SL, Rankin C. The eyes don't have it: lie detection and neuro-linguistic programming. PLoS ONE 7(7), e 40259 (2012).

13 McDonnell D. David McDonnell: time for NLP to be part of mainstream GP consultation teaching? Br. J. Gen. Pract. 64(624), 363 (2014).

- Argues that skills associated with neurolinguistic programming should be considered as part of a consultation communication framework.

14 Morley S. The self in pain. Rev. Pain 4(1), 24-27 (2010).

- Describes how pain can affect an individual's sense of self.

15 Beck RS, Daughtridge R, Sloane PD. Physician-patient communication in the primary care office: a systematic review. J. Am. Board Fam. Pract. 15(1), 25-38 (2002).

16 Matthys J, Elwyn G, Van Nuland M et al. Patients' ideas, concerns, and expectations (ICE) in general practice: impact on prescribing. Br. J. Gen. Pract. 59(558), 29-36 (2009). 